\title{
The Impact of Low-Grade Germinal Matrix-Intraventricular Hemorrhage on Neurodevelopmental Outcome of Very Preterm Infants
}

\author{
Pauline Reubsaet Annemieke J. Brouwer Ingrid C. van Haastert \\ Margaretha J. Brouwer Corine Koopman Floris Groenendaal Linda S. de Vries \\ Department of Neonatology, Wilhelmina Children's Hospital and Brain Center Rudolf Magnus, University Medical Center \\ Utrecht, Utrecht, The Netherlands
}

\section{Keywords}

Hemorrhage $\cdot$ Preterm · Ultrasound $\cdot \mathrm{MRI} \cdot$ Outcome $\cdot$ Germinal matrix-intraventricular hemorrhage $\cdot$ Cerebral palsy

\begin{abstract}
Background: Very preterm infants often show germinal matrix-intraventricular hemorrhage (GMH-IVH) on cranial ultrasound (cUS). Aim: To determine the impact of low-grade GMH-IVH on early neurodevelopmental outcome in very preterm infants. Methods: A retrospective case-control study in very preterm infants with and without low-grade GMH-IVH on cUS. Additional magnetic resonance imaging (MRI) was available in all infants with a gestational age (GA) $<28$ weeks and high-risk infants $>28$ weeks. Infants were seen at 2 years' corrected age to assess neurodevelopment. Results: In total, 136 infants (GA 24-32 weeks) with lowgrade GMH-IVH on cUS were matched with 255 controls. Outcome data was available for 342 (87\%) infants. Adverse outcome (i.e., cerebral palsy [CP], neurodevelopmental delay) was present in 11 (9\%) cases and 20 (9\%) controls. No statistically significant differences in outcome were found between cases and controls. Additional MRI was performed in 165/391 infants (42\%) and showed additional lesions in 73 (44\%) infants that could explain subsequent development of
\end{abstract}

\section{KARGER}

(C) 2017 S. Karger AG, Basel

E-Mail karger@karger.com

www.karger.com/neo
CP in 2 out of 5 infants and epilepsy in 1 of 2 infants. Conclusion: Very preterm infants with low-grade GMH-IVH on cUS have a similar early neurodevelopmental outcome compared with controls. Additional MRI showed mostly subtle abnormalities that were missed with cUS, but these could not explain subsequent development of CP and developmental delay in all infants.

\footnotetext{
(c) 2017 S. Karger AG, Basel
}

\section{Introduction}

Germinal matrix-intraventricular hemorrhage(GMHIVH) is common in preterm infants with an incidence of about $11 \%$ for low-grade GMH-IVH (grade I-II) and 3-5\% for severe GMH-IVH (grade III-IV) [1, 2]. Several studies $[3,4]$ have shown the negative impact of severe GMH-IVH on neurodevelopmental outcome, but there is no agreement about the impact of low-grade GMHIVH on outcome.

A higher risk of neurodevelopmental delay or cerebral palsy (CP) in infants with low-grade GMH-IVH compared with infants without GMH-IVH on cranial ultrasound (cUS) has been reported [5-9]. Some of these studies corrected for cUS lesions known to have an adverse effect on 
outcome (e.g., cystic periventricular leukomalacia and cerebellar lesions) [6, 7] or excluded those infants $[8,11]$.

Others did not show a difference in outcome between infants with and without low-grade GMH-IVH [10-13].

These studies used cUS to detect GMH-IVH and may have missed subtle white matter or cerebellar lesions, which also affect neurodevelopmental outcome [14-16].

To give a more definitive answer about the impact of low-grade GMH-IVH on outcome, it was previously suggested that magnetic resonance imaging (MRI) should additionally be performed [17].

MRI at term equivalent age (TEA) provides additional information on subtle white matter abnormalities and cerebellar lesions, as well as information on myelination of the posterior limb of the internal capsule, which are important for cognitive outcome and prediction of $\mathrm{CP}$ [14, 18-20].

The aim of this retrospective case-control study was to compare neonatal cUS and MRI findings and neurodevelopmental outcome at 2 years' corrected age in infants with and without low-grade GMH-IVH.

\section{Patients and Methods}

The ethics committee of the University Medical Center Utrecht waived the requirement to obtain informed consent for this retrospective study with anonymized data. This case-control study used prospectively collected data for preterm infants admitted to our level III NICU. Infants with a gestational age (GA) $\leq 32$ weeks, born between January 2008 and December 2013, with a grade I or II GMH-IVH [21] on cUS, were eligible for this study. Infants with congenital abnormalities, a grade III or IV GMH-IVH, cystic periventricular leukomalacia, cerebellar hemorrhage, or focal infarction diagnosed with cUS were excluded.

Controls without GMH-IVH on cUS were retrieved from the cUS database and matched with cases based on GA, birth weight, sex, and year of birth. Whenever possible, 2 matches were selected per case.

Maternal and perinatal data were retrieved from clinical and neuroimaging databases. Maternal educational level was defined according to the classification of the Dutch Central Bureau for Statistics [22] (Table 1).

\section{Cranial Ultrasound Examination}

cUS was performed using a Toshiba Aplio (Toshiba Medical Systems, Zoetermeer, The Netherlands) within $6 \mathrm{~h}$ after admission, on days 3 and 7, weekly until discharge, and again at TEA. Periventricular echogenicity present for more than 1 week was diagnosed as periventricular leukomalacia grade I [23]. Post-haemorrhagic ventricular dilatation (PHVD) was defined as a ventricular index $>97$ th percentile [24].

MRI Assessment

MRI, performed at 30-32 weeks' postmenstrual age and again at TEA was part of routine clinical care for infants born $<28$ weeks'
GA. Beyond 28 weeks' GA, additional MRI was performed when cUS suggested white matter abnormalities. MRI was performed on a 3.0-T MR system (Philips Healthcare, Best, The Netherlands) using an 8-channel sense head coil as reported previously. The scanning protocol included sagittal and coronal 3D-T1- and T2weighted images, and diffusion-weighted imaging. Abnormalities at TEA were evaluated using the scoring system by Kidokoro et al. [25] by 2 neonatologists (L.S.d.V., F.G.), who were blinded to the clinical outcome.

\section{Assessment of Neurodevelopmental Outcome}

Neurodevelopmental outcome was evaluated between 18 and 30 months' corrected age using the Griffiths Mental Development Scales (GMDS) or the Bayley Scales of Infant and Toddler Development 3rd Edition (BSITD-III). Mild neurodevelopmental delay was defined as a score $<88$ for the GMDS or $<85$ for the BSITD-III. Moderate neurodevelopmental delay was defined as a score of $<76$ for GMDS and $<70$ for BSITD-III. CP was defined according to criteria reported previously [26]. The severity of CP was classified according to the Gross Motor Function Classification System (GMFCS) [27].

Visual impairment was defined as a vision of $\leq 0.3$ with correction. Hearing impairment was defined as the use of hearing aids or cochlear implants. Epilepsy was defined as having seizures beyond the neonatal period requiring antiepileptic drugs.

Statistical Analysis

Statistical analysis was performed using IBM SPSS Statistics Version 21. Perinatal characteristics and outcome data of cases and controls were compared using $t$ tests and Mann-Whitney $\mathrm{U}$ tests, $\chi^{2}$ tests, or Fisher exact tests when appropriate.

Multivariable analysis was used to evaluate the influence of all factors that were significantly different between both groups at baseline on BSITD-III scores. $p<0.05$ was considered statistically significant.

\section{Results}

One hundred and thirty-six very preterm infants with low grade GMH-IVH were included and matched with 255 controls. The clinical characteristics are summarized in Table 1 . Nine of the 136 cases $(6.6 \%)$ and 11 of the 255 controls (4.3\%) died during the neonatal period.

\section{Neurodevelopmental Outcome}

Of the complete study population, 5 (3.7\%) infants with low-grade GMH-IVH and none of the controls were lost to follow-up. Twenty-two (6\%) infants, 3 (2.2\%) cases and 19 controls (7.5\%), did not complete follow-up to the age of 18-30 months' corrected age because of a favorable outcome during earlier visits. Ten infants, 3 (2.2\%) cases and 7 (2.7\%) control infants, were followed up to 18-30 months, but could not be tested due to behavioral disorders or were seen in a local hospital (Table 2). 
Table 1. Clinical characteristics

\begin{tabular}{|c|c|c|c|}
\hline Total $(n=391)$ & $\begin{array}{l}\text { Low-grade GMH-IVH } \\
(n=136)\end{array}$ & $\begin{array}{l}\text { Control group } \\
(n=255)\end{array}$ & $p$ \\
\hline \multicolumn{4}{|l|}{ Maternal characteristics } \\
\hline Maternal age at delivery, years & $31.1[6.2]$ & $31.6[7.4]$ & 0.10 \\
\hline Primipara & $95(69.9)$ & $152(59.6)$ & 0.05 \\
\hline \multicolumn{4}{|l|}{ Educational level } \\
\hline Low & $16(11.8)$ & $40(15.7)$ & 0.21 \\
\hline Middle & $25(18.4)$ & $50(19.6)$ & 0.63 \\
\hline High & $44(32.4)$ & $64(25.1)$ & 0.13 \\
\hline Missing & $51(37.5)$ & $101(39.6)$ & 0.17 \\
\hline \multicolumn{4}{|l|}{ Perinatal characteristics } \\
\hline \multicolumn{4}{|l|}{ Delivery } \\
\hline Spontaneous vaginal delivery & $80(58.8)$ & $102(40.0)$ & $<0.001^{*}$ \\
\hline Breech delivery & $10(7.4)$ & $14(5.5)$ & 0.47 \\
\hline Assisted vaginal delivery & $3(2.2)$ & $0(0.0)$ & $0.02 *$ \\
\hline Caesarean section & $43(31.6)$ & $139(54.5)$ & $<0.001^{*}$ \\
\hline Multiple gestation & $43(31.6)$ & $94(36.9)$ & 0.3 \\
\hline Antenatal corticosteroid use & $69(50.7)$ & $183(71.8)$ & $<0.001^{*}$ \\
\hline \multicolumn{4}{|l|}{ Apgar score $(n=387)$} \\
\hline $1 \mathrm{~min}$ & $6(4)$ & $7(3)$ & $0.007^{*}$ \\
\hline $5 \mathrm{~min}$ & $8(2)$ & $8(2)$ & 0.07 \\
\hline \multicolumn{4}{|l|}{ Neonatal characteristics } \\
\hline Female & $65(47.8)$ & $126(49.4)$ & 0.76 \\
\hline Gestational age at birth, weeks & $28.4(3.9)$ & $28.7(3.0)$ & 0.12 \\
\hline Gestational age $<28$ weeks & $58(42.6)$ & $90(35.3)$ & 0.15 \\
\hline Birth weight, $g$ & $1,125[510]$ & $1,170[430]$ & 0.54 \\
\hline Mechanical ventilation $\geq 1$ week & $53(39.0)$ & $51(20.0)$ & $<0.001^{*}$ \\
\hline Hypotension & $75(55.1)$ & $99(38.8)$ & $0.002^{*}$ \\
\hline Additional fluid administration & $25(18.4)$ & $40(15.7)$ & 0.50 \\
\hline Inotropics & $50(36.8)$ & $59(23.1)$ & $0.004^{*}$ \\
\hline Patent ductus arteriosus & $43(31.6)$ & $56(22.0)$ & $0.04^{*}$ \\
\hline Necrotizing enterocolitis & $7(5.3)$ & $7(2.8)$ & 0.21 \\
\hline Early-onset sepsis & $1(0.7)$ & $3(1.2)$ & 0.68 \\
\hline Late-onset sepsis & $30(22.1)$ & $57(22.4)$ & 0.95 \\
\hline Neonatal surgery & $28(20.6)$ & $24(9.4)$ & $0.002^{*}$ \\
\hline Bilateral GMH-IVH & $40(10.2)$ & - & - \\
\hline PHVD & $10(2.6)$ & - & - \\
\hline Neonatal death & $9(6.6)$ & $11(4.3)$ & 0.33 \\
\hline
\end{tabular}

Data are presented as medians [interquartile range] or $n(\%)$. GMH-IVH, germinal matrix-intraventricular hemorrhage; PHVD, post-haemorrhagic ventricular dilatation. * Statistically significant differences.

Visual impairment occurring in 3 infants consisted of severe retinopathy of prematurity in 1 case and cortical visual impairment in 1 case and 1 control. Hearing impairment was caused by abnormalities in the middle ear $(n=1)$ or cochlea $(n=3)$.

No statistically significant differences between cases and controls were found for developmental delay or any form of impairment. However, when looking at the sub-

Low-Grade IVH and Neurodevelopment scales of the BSITD-III, infants in the GMH-IVH group obtained a significantly higher score on cognition than controls ( $p=0.04$ and $p=0.02$, corrected and not-corrected for prematurity, respectively), but there were no significant differences in motor scores (Table 2). Following multivariable analysis, adjusting for GA and maternal educational level, there was no longer a significant difference between the 2 groups. Furthermore, the interaction

Neonatology 2017;112:203-210 DOI: $10.1159 / 000472246$ 
Table 2. Neurodevelopmental outcome

\begin{tabular}{|c|c|c|c|}
\hline $\begin{array}{l}\text { Total } \\
(n=342)\end{array}$ & $\begin{array}{l}\text { Low-grade GMH-IVH } \\
(n=119)\end{array}$ & $\begin{array}{l}\text { Control group } \\
(n=223)\end{array}$ & $p$ \\
\hline Mild developmental delay (<1 SD) & $3(2.5)$ & $10(4.5)$ & 0.37 \\
\hline Moderate developmental delay $(<2 \mathrm{SD})$ & $2(1.7)$ & $2(0.9)$ & 0.61 \\
\hline Cerebral palsy & $2(1.7)$ & $3(1.3)$ & 1.0 \\
\hline Visual impairment & $2(1.7)$ & $1(0.4)$ & 1.0 \\
\hline Hearing impairment & $1(0.8)$ & $3(1.3)$ & 1.0 \\
\hline Epilepsy & $1(0.8)$ & $1(0.4)$ & 1.0 \\
\hline BSITD-III $(n=244)$ & $(n=85)$ & $(n=159)$ & \\
\hline \multicolumn{4}{|l|}{ Corrected age } \\
\hline Cognitive composite score & $105[13]$ & $105[15]$ & $0.04^{*}$ \\
\hline Motor composite score & $110[14]$ & $110[15]$ & 0.61 \\
\hline Fine motor function & $13[3]$ & $13[4]$ & 0.37 \\
\hline Gross motor function & $9[1]$ & $9[2]$ & 0.60 \\
\hline \multicolumn{4}{|l|}{ Chronological age } \\
\hline Cognitive composite score & $100[13]$ & $95[10]$ & $0.02 *$ \\
\hline Motor composite score & $100[9]$ & $100[9]$ & 0.94 \\
\hline Fine motor function & $11[2]$ & $11[2]$ & 0.71 \\
\hline Gross motor function & $8[1]$ & $8[2]$ & 0.58 \\
\hline GMDS $(n=87)$ & $(n=31)$ & $(n=56)$ & \\
\hline DQ for corrected age & $99.0[10.0]$ & $99.5[12.8]$ & 0.82 \\
\hline DQ for chronological age & $89.0[8.0]$ & $89.5[12.0]$ & 0.92 \\
\hline
\end{tabular}

Data are presented as medians [interquartile range] or $n$ (\%). GMH-IVH, germinal matrix-intraventricular hemorrhage; BSITD-III, Bayley Scales of Infant and Toddler Development 3rd Edition; SD, standard deviation, DQ, developmental quotient; GMDS, Griffiths Mental Development Scales. * Statistically significant.

between maternal educational level and GA $(<28$ weeks or $\geq 28$ weeks) was significantly associated $(p<0.001)$ with outcome (Fig. 1).

As MRI identified low-grade GMH-IVH in 13 infants without cUS abnormalities (see below), the analysis was repeated after removing these infants from the controls and adding them to the low-grade GMH-IVH group. These results did not change our findings.

\section{Neuroimaging}

Magnetic Resonance Imaging

In total, 69/136 (50.7\%) cases and 96/255 (37.6\%) controls additionally had an MRI. MRI was performed in 51 cases and 79 controls as routine clinical care because of GA $<28$ weeks, and in 18 cases and 17 controls because of inhomogeneous echogenicity on cUS.

Infants with low-grade GMH-IVH scored significantly higher than controls when looking at the Kidokoro global abnormality score, white matter score, and cerebellar score $(p<0.01)$ [25] (Table 3$)$.
Additional Value of Magnetic Resonance Imaging

MRI identified additional abnormalities in $37 / 69$ (53.6\%) cases and 36/96 (37.5\%) controls. Low-grade GMH-IVH was detected on MRI only in $13 / 96(13.5 \%)$ controls.

White matter lesions were noted in $27 / 69$ (39.1\%) cases and 19/96 (19.8\%) controls $(p<0.01)$, and punctate cerebellar lesions in 15/69 (21.7\%) cases and 7/96 (7.3\%) controls $(p<0.01)$. In one of the cases, cerebellar atrophy due to a large unilateral cerebellar hemorrhage was first detected on TEA MRI.

Other lesions seen on MRI, but missed on pre-MRI cUS, consisted of a focal infarction ( $n=1)$ (Fig. 2), asymmetrical myelination of the posterior limb of the internal capsule $(n=1)$, and type I Arnold-Chiari malformation $(n=1)$.

MRI at TEA was available in 3 of the 4 infants with a moderate developmental delay and in 9 of the 13 infants with a mild delay. No additional abnormalities were found on MRI in these infants. 


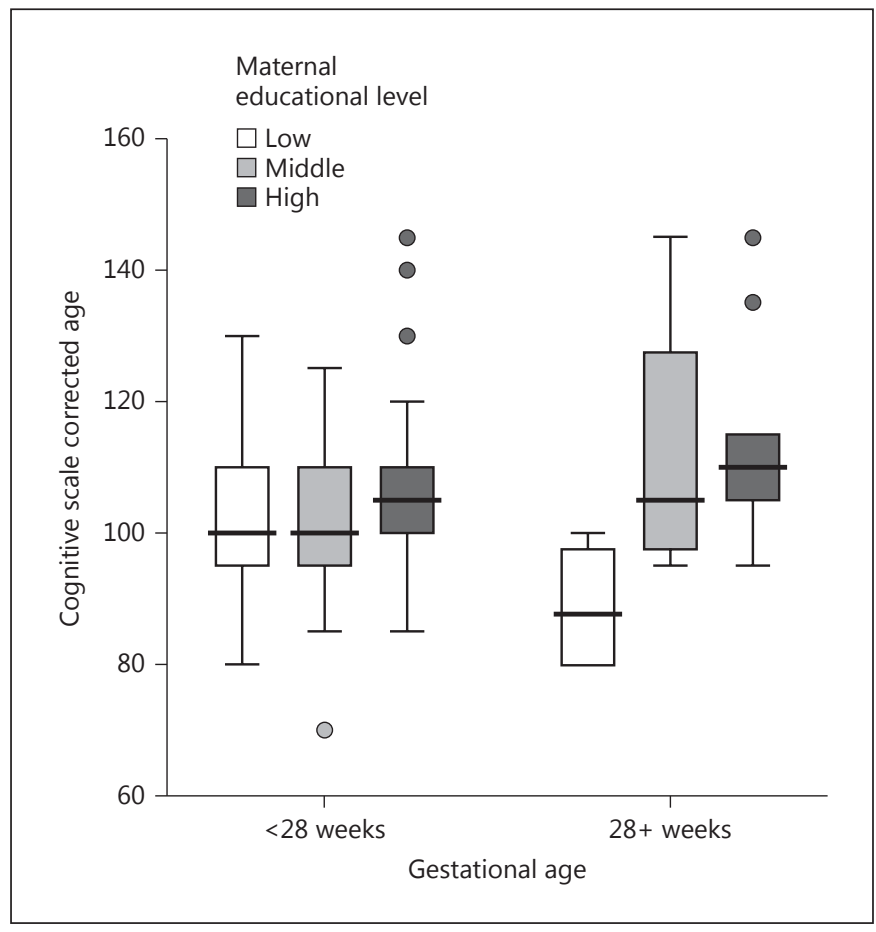

Fig. 1. Cognition composite score at corrected age divided according to maternal educational level.

Of the 5 infants with CP (2 with GMH-IVH, 3 controls), 2 had routine MRI at TEA and 2 had it during infancy following the diagnosis of CP. In one infant, who developed PHVD after a grade II GMH-IVH, MRI additionally showed a small unilateral cerebellar hemorrhage, which could not explain his mild spastic diplegia. The second infant, one of monochorionic twins, with a bilateral GMH, developed a bilateral spastic CP (GMFCS level I). MRI following this diagnosis provided additional information, showing periventricular gliosis.

MRI was performed in 2 of 3 controls with CP. In one infant, TEA MRI showed unexplained asymmetric myelination of the posterior limb of the internal capsule that could explain subsequent development of unilateral spastic CP. In the other control infant who had an MRI in infancy, no abnormalities were found.

Both infants who developed epilepsy during follow-up had a TEA MRI. In one infant with unilateral GMH on cUS, MRI at TEA showed a subcortical cyst as a possible explanation for developing epilepsy (Fig. 3). These additional lesions were seen on cUS after the MRI diagnosis. In the other infant, MRI additionally showed bilateral GMH-IVH, not seen with cUS.

Low-Grade IVH and Neurodevelopment
Table 3. Additional MRI findings using visual assessment and the Kidokoro score

\begin{tabular}{|c|c|c|c|}
\hline Total $(n=165)$ & $\begin{array}{l}\text { Low grade } \\
\text { GMH-IVH } \\
(n=69)\end{array}$ & $\begin{array}{l}\text { Control } \\
\text { group } \\
(n=96)\end{array}$ & $p$ \\
\hline Females & $29(42.0)$ & $48(50.0)$ & 0.31 \\
\hline Gestational age at birth, weeks & $26.3[2.6]$ & $26.7[1.8]$ & 0.82 \\
\hline Birth weight, $g$ & $910[375]$ & $923[293]$ & 0.69 \\
\hline \multicolumn{4}{|l|}{ Age at MRI } \\
\hline Term equivalent age & $63[91.3]$ & $90[93.8]$ & 0.55 \\
\hline Only at 30-34 weeks' CA & $4[5.8]$ & $3[3.1]$ & 0.40 \\
\hline Beyond neonatal period & $2[2.9]$ & $3[3.1]$ & 0.93 \\
\hline Kidokoro total score $(n=153)$ & $5(3)$ & $3(3)$ & $<0.001^{*}$ \\
\hline White matter score & $3(2)$ & $3(2)$ & $0.001^{*}$ \\
\hline Cortical grey matter score & $1(1)$ & $1(1)$ & 0.46 \\
\hline Deep grey matter score & $0(0)$ & $0(0)$ & 0.07 \\
\hline Cerebellar score & $0(1)$ & $0(1)$ & $0.005^{*}$ \\
\hline Additional abnormalities on MRI & $37(53.6)$ & $36(37.5)$ & $0.04^{*}$ \\
\hline GMH-IVH & - & $13(13.5)$ & - \\
\hline White matter lesions & $27(39.1)$ & $19(19.8)$ & $0.006^{*}$ \\
\hline Focal punctate & $23(33.3)$ & $16(16.7)$ & $0.01^{*}$ \\
\hline Extensive punctate & $3(4.3)$ & $1(1.0)$ & 0.31 \\
\hline Linear & $1(1.4)$ & $1(1.0)$ & 1.0 \\
\hline Cystic lesions & $1(1.4)^{\mathrm{a}}$ & $1(1.0)^{\mathrm{b}}$ & 1.0 \\
\hline Cerebellar lesions & $15(21.7)$ & $7(7.3)$ & $0.007^{*}$ \\
\hline Punctate unilateral & $10(14.5)$ & $6(6.2)$ & 0.08 \\
\hline Punctate bilateral & $4(5.8)$ & $1(1.0)$ & 0.16 \\
\hline Extensive unilateral & $1(1.4)$ & 0 & 0.42 \\
\hline Other & $4(5.87)$ & $2(2.1)$ & 0.21 \\
\hline
\end{tabular}

Data are presented as medians [interquartile range] or $n(\%)$. GMH-IVH, germinal matrix-intraventricular hemorrhage; CA, corrected age. ${ }^{*}$ Statistically significant differences. ${ }^{\text {a }}$ Single subcortical cyst (Fig. 3a, b). ${ }^{\text {b }}$ This cystic lesion was a focal infarct (Fig. 2a, b).

In all infants with a visual impairment, no abnormalities on MRI could be found. In 2 of the 4 infants with a hearing impairment, MRI was performed and was normal.

An overview of additional abnormalities in infants with adverse outcome is presented in online supplementary Table 1 (see www.karger.com/doi/10.1159/000472246).

\section{Discussion}

In this large hospital-based case-control study, no differences in neurodevelopmental outcome or any form of impairment was found between very preterm infants with low-grade GMH-IVH, diagnosed with cUS, and matched controls. The overall incidence of impairment or developmental delay was low. It was of interest that many of

Neonatology 2017;112:203-210 207 
Fig. 2. a Large unilateral hemorrhagic watershed infarct on axial T2-weighted MRI. b Cranial ultrasound performed after MRI also shows the lesion as an area of increased echogenicity.

Fig. 3. Coronal term equivalent age MRI, T2-weighted sequence (a), showing bilateral subependymal pseudocysts, also seen with cranial ultrasound (b) and a subcortical cyst in the right frontal lobe with a linear line of low signal intensity. Following the magnetic resonance diagnosis, the cyst and linear echogenic line were also seen with a high-resolution linear transducer. The infant developed postneonatal epilepsy at 7 months of age requiring antiepileptic medication, but had no further seizures and had a developmental quotient of 99 uncorrected and 107 corrected age at 24 months. $\mathrm{CMV}$ and metabolic investigations were negative.
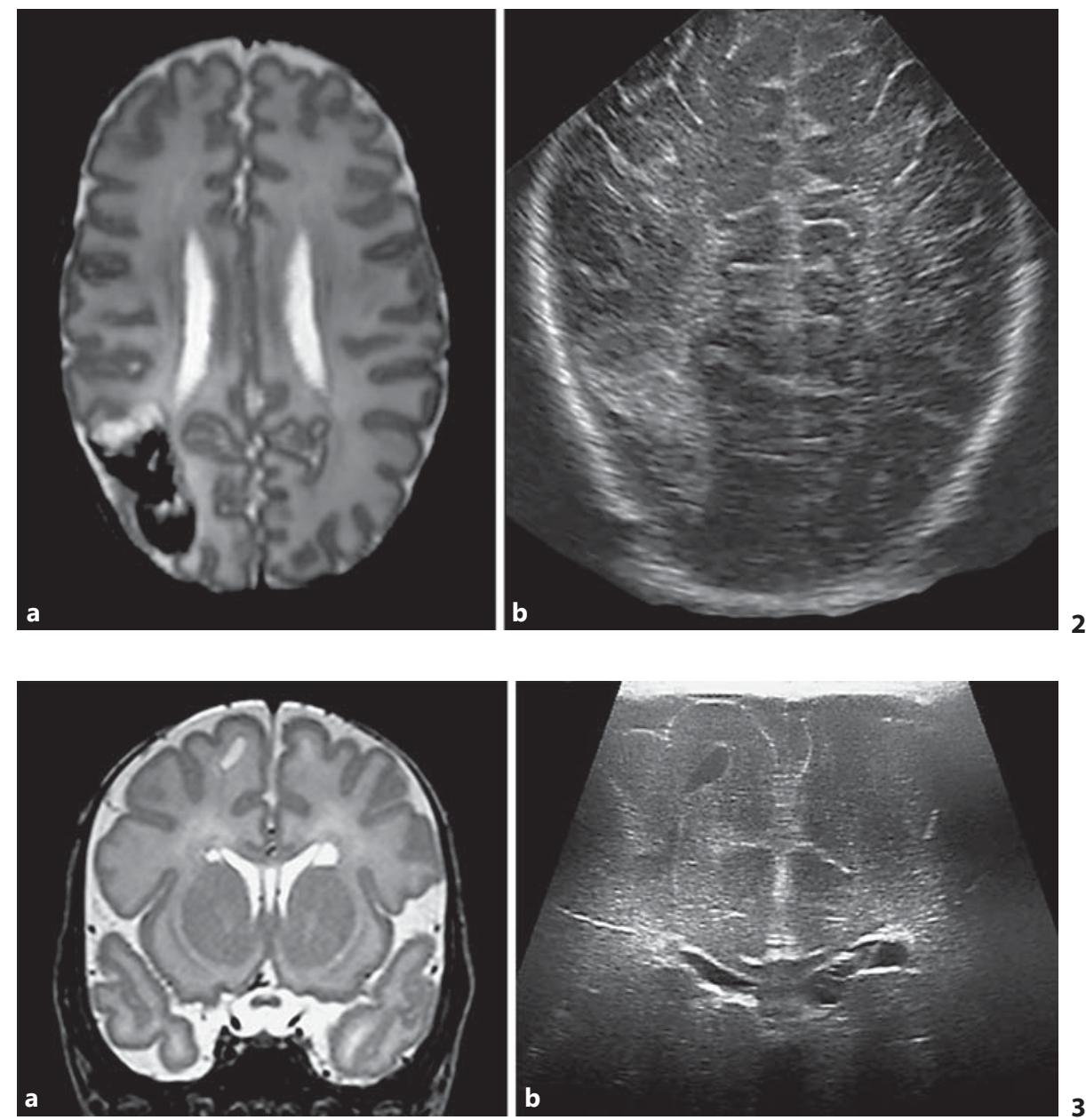

the known risk factors for GMH-IVH were also present in this low-grade hemorrhage group $[1,2]$.

To the best of our knowledge, this is the first study with additional MRIs, performed in a subgroup of the study population. Additional lesions were seen on MRI in 54\% of the infants with low-grade GMH-IVH, and $38 \%$ of those with normal cUS findings. It is important to note that low-grade GMH-IVH was found with MRI in 5\% of the controls. MRI abnormalities consisted mostly of subtle white matter abnormalities and punctate cerebellar lesions. In 2 of the 5 infants these additional lesions were a likely explanation for subsequent development of CP or postneonatal epilepsy.

No statistically significant differences were found between infants with and without low-grade GMH-IVH after multivariable analysis. Only maternal education and GA and their interaction were significantly associated with cognitive outcome.
Several previous studies reported a significantly worse outcome in infants with low-grade GMH-IVH $[6,7,9]$. Klebermass-Schrehof et al. [8] compared very preterm infants with and without an IVH at 5 years of age. They found a significantly higher percentage of CP in infants with a grade I GMH (22.2\%) and a grade II IVH (47.4\%) compared with infants without GMH-IVH. Large differences existed in baseline characteristics between the infants with and without GMH-IVH. Bolisetty et al. [7] and Patra et al. [11] also found a significantly higher rate of impairment in infants with low-grade GMH-IVH when compared to infants without GMH-IVH. Both studies took other cerebral lesions, diagnosed with cUS, into account when analyzing their results. As these infants did not get an MRI, it is possible that additional important cerebral lesions were missed in some infants. 
In agreement with our study, several studies did not find any differences in outcome between infants with and without low-grade GMH-IVH $[9,10,12,13]$.

The EPIPAGE Study Group only found a significantly higher odds ratio for severe cognitive impairment in infants with a grade II IVH compared with infants without an IVH, but no other differences between infants with a grade I or II IVH compared with infants without GMHIVH. They also reported that the most important predictor for cognitive impairment was socioeconomic status and maternal educational level [8].

Recently, Radic et al. [16] found no significant differences between infants with a grade I GMH or no GMHIVH at the age of 2-3 years. In infants with a grade II GMH-IVH, a significantly higher rate of mild CP was found.

A major strength of our study is that additional MRI was performed in 165 infants and that abnormalities were evaluated with a standardized and validated TEA MRI scoring system [25]. In particular, subtle white matter lesions and punctate cerebellar lesions were missed with cUS. The effect of these lesions on long-term outcome still needs to be determined. Additional lesions diagnosed with MRI could explain neurodevelopmental impairment in a few infants. Another study looked at MRI abnormalities in infants with low-grade GMH-IVH, but did not describe the effect of these abnormalities on neurodevelopmental outcome [28]. A study conducted by Vasileiadis et al. [29] showed reduced cortical grey matter volume in infants with low-grade GMH-IVH and GA $<30$ weeks compared with controls. Another study by Morita et al. [30] used diffusion tensor imaging to determine differences in the development of white matter tracts between preterm infants with and without low-grade GMHIVH in infants $<30$ weeks' gestation. They found lower fractional anisotropy values in the superior cerebellar peduncle and in the motor tracts, but did not provide any outcome data.

There are also several limitations that need to be addressed. First of all, the retrospective design and the fact that cUS was performed by many clinicians with differences in experience. The mastoid window was not consistently used during the first years of the study period. cUS images were reviewed by an experienced neonatologist when patient files and databases provided contradictory information about the presence of GMH-IVH, but in several cases images were of poor quality and therefore difficult to grade retrospectively. The number of infants was relatively small in each group compared to several previous studies $[8,11]$. Although a few studies with a similar

Low-Grade IVH and Neurodevelopment group size did find a small difference in outcome between infants with and without GMH-IVH, it is possible that we would have found differences with a larger group size. However, in both studies, outcome was more adverse than in our population with a higher percentage of low MDI and PDI, and more CP. In addition, MRI had not been used in these 2 studies, and parenchymal involvement may have been missed. In one study [11], GA and in particular BW were lower than in our cohort, which may have contributed to their adverse outcome. Not all infants in our study had an MRI, and we cannot exclude that we would have been able to show a relation between MRI findings and neurodevelopmental outcome, as MRI shows the full extent of brain lesions. Finally, the limited follow-up period up to 2 years' corrected age was another limitation of this study. Subtle cognitive problems might not be apparent at this age, as reported Vohr et al. [6] who found no differences in outcome between infants with low-grade GMH-IVH and without GMH-IVH at 3-5 years of age [14], but a significant difference was seen at 16 years of age [16]. Follow-up should therefore continue to school age to show the full effect of low-grade GMHIVH on long-term neurodevelopmental outcome.

\section{Conclusion}

Preterm infants with low-grade GMH-IVH on cUS demonstrated a similar early neurodevelopmental outcome compared with controls without GMH-IVH. Additional neuroimaging with MRI showed additional abnormalities in more than half of the infants with lowgrade GMH-IVH, which would support routine use of MRI at TEA. These mostly subtle white matter abnormalities, however, could only provide an explanation for $\mathrm{CP}$ and developmental delay in a minority of the infants. Further research is needed to determine the effect of lowgrade GMH-IVH on long-term outcome.

\section{Disclosure Statement}

The authors declare no conflicts of interest. 


\section{References}

1 Larroque B, Marret S, Ancel PY, Arnaud C, Marpeau L, Supernant K, Pierrat V, Rozé JC, Matis J, Cambonie G, Burguet A, Andre M, Kaminski M, Bréart G; EPIPAGE Study Group: White matter damage and intraventricular hemorrhage in very preterm infants: the EPIPAGE study. J Pediatr 2003;143:477483.

2 Cust AE, Darlow BA, Donoghue DA; Australian and New Zealand Neonatal Network (ANZNN): Outcomes for high risk New Zealand newborn infants in 1998-1999: a population based, national study. Arch Dis Child Fetal Neonatal Ed 2003;88:F15-F22.

3 Brouwer A, Groenendaal F, van Haastert IL, Rademaker K, Hanlo P, de Vries L: Neurodevelopmental outcome of preterm infants with severe intraventricular hemorrhage and therapy for post-hemorrhagic ventricular dilatation. J Pediatr 2008;152:648-654.

4 Futagi Y, Toribe Y, Ogawa K, Suzuki Y: Neurodevelopmental outcome in children with intraventricular hemorrhage. Pediatr Neurol 2006;34:219-224.

5 Adams-Chapman I, Hansen NI, Stoll BJ, Higgins R; NICHD Research Network: Neurodevelopmental outcome of extremely low birth weight infants with posthemorrhagic hydrocephalus requiring shunt insertion. Pediatrics 2008;121:e1167-e1177.

6 Vohr BR, Allan W, Katz KH, Schneider K, Tucker R, Ment LR: Adolescents born prematurely with isolated grade 2 haemorrhage in the early 1990s face increased risks of learning challenges. Acta Paediatr 2014;103:10661071.

7 Bolisetty S, Dhawan A, Abdel-Latif M, Bajuk B, Stack J, Lui K; New South Wales and Australian Capital Territory Neonatal Intensive Care Units' Data Collection: Intraventricular hemorrhage and neurodevelopmental outcomes in extreme preterm infants. Pediatrics 2014;133:55-62.

8 Klebermass-Schrehof K, Czaba C, Olischar M, Fuiko R, Waldhoer T, Rona Z, Pollak A Weninger M: Impact of low-grade intraventricular hemorrhage on long-term neurodevelopmental outcome in preterm infants. Childs Nerv Syst 2012;28:2085-2092.

9 Beaino G, Khoshnood B, Kaminski M, Marret S, Pierrat V, Vieux R, Thiriez G, Matis J, Picaud JC, Rozé JC, Alberge C, Larroque B, Bréart G, Ancel PY; EPIPAGE Study Group: Predictors of the risk of cognitive deficiency in very preterm infants: the EPIPAGE prospective cohort. Acta Paediatr 2011;100:370378.
10 Vavasseur C, Slevin M, Donoghue V, Murphy JF: Effect of low grade intraventricular hemorrhage on developmental outcome of preterm infants. J Pediatr 2007; 151:e6; author reply e6-e7.

11 Patra K, Wilson-Costello D, Taylor HG, Mercuri-Minich N, Hack M: Grades I-II intraventricular hemorrhage in extremely low birth weight infants: effects on neurodevelopment. J Pediatr 2006;149:169-173.

12 Payne AH, Hintz SR, Hibbs AM, Walsh MC, Vohr BR, Bann CM, Wilson-Costello DE; Eunice Kennedy Shriver National Institute of Child Health and Human Development Neonatal Research Network: Neurodevelopmental outcomes of extremely low-gestationalage neonates with low-grade periventricularintraventricular hemorrhage. JAMA Pediatr 2013;167:451-459.

13 Ross G, Boatright S, Auld PA, Nass R: Specific cognitive abilities in 2-year-old children with subependymal and mild intraventricular hemorrhage. Brain Cogn 1996;32:1-13.

14 Vohr B, Garcia Coll C, Flanagan P, Oh W: Effects of intraventricular hemorrhage and socioeconomic status on perceptual, cognitive, and neurologic status of low birth weight infants at 5 years of age. J Pediatr 1992;121:280 285.

15 Sherlock RL, Anderson PJ, Doyle LW; Victorian Infant Collaborative Study Group: Neurodevelopmental sequelae of intraventricular haemorrhage at 8 years of age in a regional cohort of ELBW/very preterm infants. Early Hum Dev 2005;81:909-916.

16 Radic JA, Vincer M, McNeely PD: Outcomes of intraventricular hemorrhage and posthemorrhagic hydrocephalus in a populationbased cohort of very preterm infants born to residents of Nova Scotia from 1993 to 2010 J Neurosurg Pediatr 2015;15:580-588.

17 Hintz SR, Slovis T, Bulas D, Van Meurs KP, Perritt R, Stevenson DK, Poole WK, Das A, Higgins RD; NICHD Neonatal Research Network: Interobserver reliability and accuracy of cranial ultrasound scanning interpretation in premature infants. J Pediatr 2007; 150:592596, 596.e1-e5.

18 Inder TE: Neurodevelopmental impact of low-grade intraventricular hemorrhage in very preterm infants. J Pediatr 2006; 149:152154.

19 Maalouf EF, Duggan PJ, Counsell SJ, Rutherford MA, Cowan F, Azzopardi D, Edwards $\mathrm{AD}$ : Comparison of findings on cranial ultrasound and magnetic resonance imaging in preterm infants. Pediatrics 2001;107:719727
20 Inder TE, Anderson NJ, Spencer C, Wells S, Volpe JJ: White matter injury in the premature infant: a comparison between serial cranial sonographic and MR findings at term. AJNR Am J Neuroradiol 2003;24:805-809.

21 Jeon TY, Kim JH, Yoo SY, Eo H, Kwon JY, Lee J, Lee M, Chang YS, Park WS: Neurodevelopmental outcomes in preterm infants: comparison of infants with and without diffuse excessive high signal intensity on MR images at near-term-equivalent age. Radiology 2012; 263:518-526.

22 Papile LA, Burstein J, Burstein R, Koffler H: Incidence and evolution of subependymal and intraventricular hemorrhage: a study of infants with birth weights less than $1,500 \mathrm{gm}$. J Pediatr 1978;92:529-534.

23 Statistics Netherlands (CBS) Standaard onderwijsindeling 2006, Edition 2014/15. http://www.cbs.nl/nl-NL/menu/methoden/ classificaties/overzicht/soi/2006/default.htm.

24 de Vries LS, Eken P, Dubowitz LM: The spectrum of leukomalacia using cranial ultrasound. Behav Brain Res 1992;49:1-6.

25 Levene MI: Measurement of the growth of the lateral ventricles in preterm infants with realtime ultrasound. Arch Dis Child 1981;56 900-904.

26 Kidokoro H, Neil JJ, Inder TE: New MR imaging assessment tool to define brain abnormalities in very preterm infants at term. AJNR Am J Neuroradiol 2013;34:2208-2214.

27 Rosenbaum P, Paneth N, Leviton A, Goldstein M, Bax M, Damiano D, Dan B, Jacobsson $B$ : A report: the definition and classification of cerebral palsy. April 2006. Dev Med Child Neurol Suppl 2007;109:8-14.

28 Palisano RJ, Cameron D, Rosenbaum PL, Walter SD, Russell D: Stability of the gross motor function classification system. Dev Med Child Neurol 2006;48:424-428.

29 Vasileiadis GT, Gelman N, Han VK, Williams LA, Mann R, Bureau Y, Thompson RT: Uncomplicated intraventricular hemorrhage is followed by reduced cortical volume at nearterm age. Pediatrics 2004; 114:e367-e372.

30 Morita T, Morimoto M, Yamada K, Hasegawa T, Morioka S, Kidowaki S, Moroto M, Yamashita S, Maeda H, Chiyonobu T, Tokuda S, Hosoi $\mathrm{H}$ : Low-grade intraventricular hemorrhage disrupts cerebellar white matter in preterm infants: evidence from diffusion tensor imaging. Neuroradiology 2015;57:507-514. 